\title{
Archéopages
}

Archéopages

Archéologie et société

41 | 10/2014-01/2015

La place du cheval

\section{La pratique urbaine de l'équarrissage à la charnière du Moyen Âge et de l'époque moderne}

L'exemple d'Elbeuf

Urban slaughter practices at the turn of the Middle Ages to the Early Modern period. The example of Elbeuf

La práctica urbana del descuartizamiento en el período de transición entre la Edad Media y la época moderna. El ejemplo de Elbeuf

\section{Maude Barme et Benoît Clavel}

\section{OpenEdition}

Journals

Édition électronique

URL : https://journals.openedition.org/archeopages/965

DOI : 10.4000/archeopages.965

ISSN : 2269-9872

Éditeur

INRAP - Institut national de recherches archéologiques préventives

Édition imprimée

Date de publication : 1 août 2015

Pagination : 30-39

ISSN : 1622-8545

Référence électronique

Maude Barme et Benoît Clavel, « La pratique urbaine de l'équarrissage à la charnière du Moyen Âge et de l'époque moderne », Archéopages [En ligne], 41 | 10/2014-01/2015, mis en ligne le 01 janvier 2017, consulté le 22 janvier 2022. URL : http://journals.openedition.org/archeopages/965 ; DOI : https:// doi.org/10.4000/archeopages.965 


\title{
La pratique urbaine de l'équarrissage à la charnière du Moyen Âge et de l'époque moderne L'exemple d'Elbeuf
}

\author{
Maude Barme CRAVO \\ Benoît Clavel CNRS, UMR 7209 «Archéozologie, Archéobotanique: sociétés, pratiques et environnements»
}

\section{0}

1. Opération menée au 57 rue Guynemer à Elbeuf, en 2013, sous la responsabilité de Bénédicte Guillot, Inrap. 2. Fouille préventive menée en 2007 sur le « Parking Hôtel-de-Ville » d'Évreux sous la responsabilité de Bénédicte Guillot, Inrap.
Le devenir des carcasses d'équidés dans les villes médiévales et modernes, et plus généralement de tout cadavre de grand mammifère, soulève deux difficultés majeures. La première tient évidemment à la nécessité de libérer l'espace intra muros de ces encombrants déchets. La seconde est liée aux désagréments accompagnant toute valorisation de ces éléments, à savoir la souillure du lieu de traitement et l'odeur nauséabonde qui s'en dégage. La présente étude se concentre sur un petit lot d'ossements d'équidés, mis en place dans la seconde moitié du XVI ${ }^{\mathrm{e}}$ siècle, à Elbeuf (Seine-Maritime) ${ }^{\mathbf{1}}$ (Guillot, à paraître). Les modalités de dépôts trahissent quelques singularités, engageant à s'interroger sur les contours de l'activité d'équarrissage à la Renaissance et sa perception par les contemporains. La réflexion sur les lieux et les contextes de pratique de l'équarrissage aux époques médiévale et moderne a d'ailleurs déjà été développée récemment (Poplin, 2013).

Confrontés à des carcasses impropres à la consommation, les citadins oscillent entre deux attitudes, la première consistant à trouver une parcelle de terrain inoccupée, au sein même des quartiers d'habitation, pour procéder à leur enfouissement. Cette solution de fortune ne s'applique cependant quà des animaux isolés et intervient de façon ponctuelle. Aussi, les fosses individuelles mises en évidence en contexte urbain relèvent plus vraisemblablement d'une initiative privée. S'agissant du bétail, cet usage s'illustre notamment sur le site d'Amiens « Avenue Charles de Gaulle » (Clavel, Robin, 2012) : plusieurs structures creusées dans les faubourgs de la ville contenaient des squelettes en connexion (porcs, mouton, vache), témoins des aléas de l'élevage et du phénomène de réforme. Quant aux équidés, la découverte d'individus esseulés reste relativement rare et les carcasses ne sont alors généralement pas mises en valeur. Ces cas sont, encore une fois, plutôt le fait de particuliers, reléguant la dépouille de leur cheval dans une fosse de fond de jardin.

La seconde option s'impose quand plusieurs bêtes sont impliquées et que leur exploitation est alors le plus souvent envisagée : elle aboutit à la constitution de dépôts d'équarrissage urbains, régulièrement localisés aux marges de la ville. Ces derniers tendent à se multiplier au cours du bas Moyen Âge et de la période moderne, comme l'atteste la quinzaine d'occurrences recensées sur le territoire métropolitain (Rodet-Belarbi, Jouanin, Clavel, à paraître) [ill. 1]. Cinq ensembles issus de ce corpus se prêtent particulièrement à une comparaison au dépôt elbeuvien, en considération de leur proximité géographique et de leur relative contemporanéité. Ces dépôts d'équarrissage se trouvent, pour trois d'entre eux, à Beauvais (Oise) (Arbogast et al., 2002 ; Lepetz, 1991). Ils se distribuent entre les sites du couvent des Cordeliers ( $\mathrm{XV}^{\mathrm{e}}$ siècle), de la rue des Jacobins $\left(\mathrm{XIV}^{\mathrm{e}}\right.$ siècle) et de l'Hôtel-Dieu ( $\mathrm{XV}^{\mathrm{e}}-\mathrm{XVII}{ }^{\mathrm{e}}$ siècle). Les deux derniers assemblages proviennent de deux fosses contigües reconnues à Évreux (Eure) ${ }^{2}$ (XVII ${ }^{\mathrm{e}}$ siècle, Clavel, inédit). Enfin, le dépôt mis au jour au cours des fouilles des Jardins du Carrousel (Paris, $\mathrm{XV}^{\mathrm{e}}-\mathrm{XVII}^{\mathrm{e}}$ siècle), plus éloigné d'Elbeuf mais présentant des similitudes en termes contextuels, sera brièvement évoqué (Rodet-Belarbi, 1993).

\section{L'équarrissage à l'aune des observations archéologiques}

De prime abord, les modalités de dépôt des restes d'équidés découverts à Elbeuf se distinguent 
1

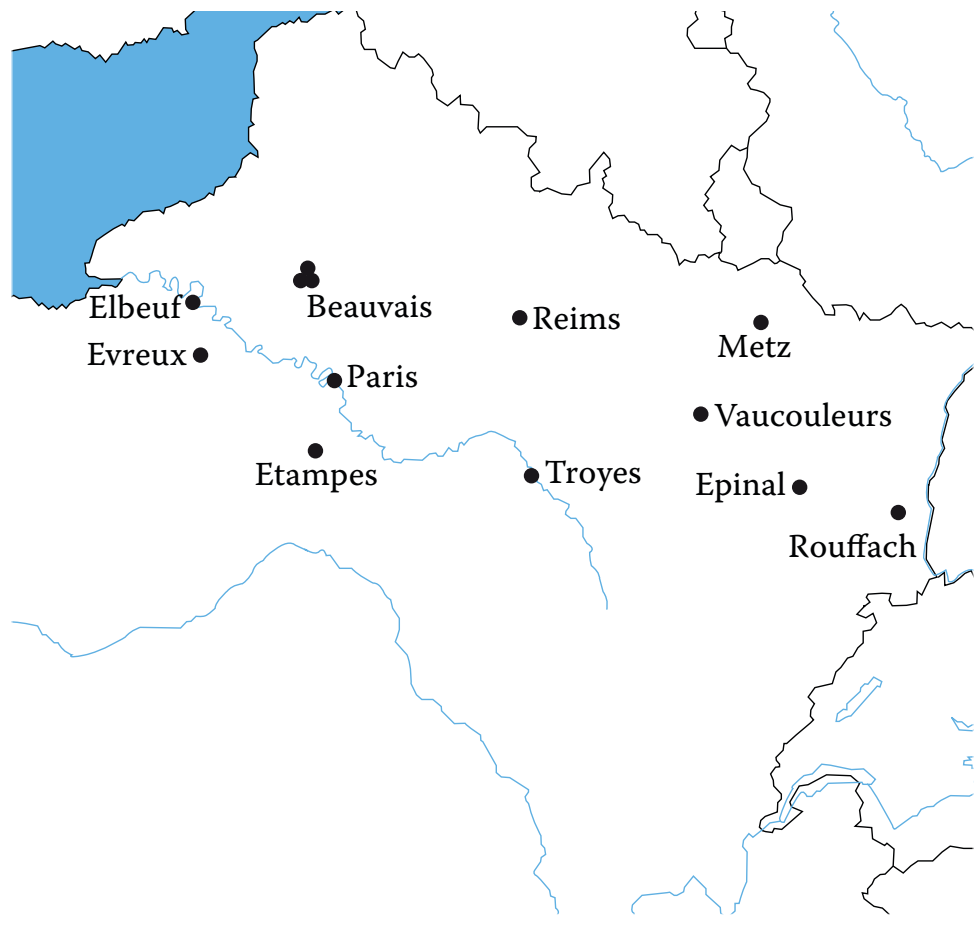

1. La majorité des dépôts 2. La fosse d'équarrissage

d'équarrissage recensés (F 369) mise au jour sur

pour la fin du Moyen Âge le site d'Elbeuf a été

et l'époque moderne installée entre un édifice

se situent en France

septentrionale.

vraisemblablement à

usage d'habitation, et

un bâtiment rectangulaire

au sud, dont le statut

s'apparenterait plutôt

à celui d'une dépendance

(réserve, étable, écurie).

cave
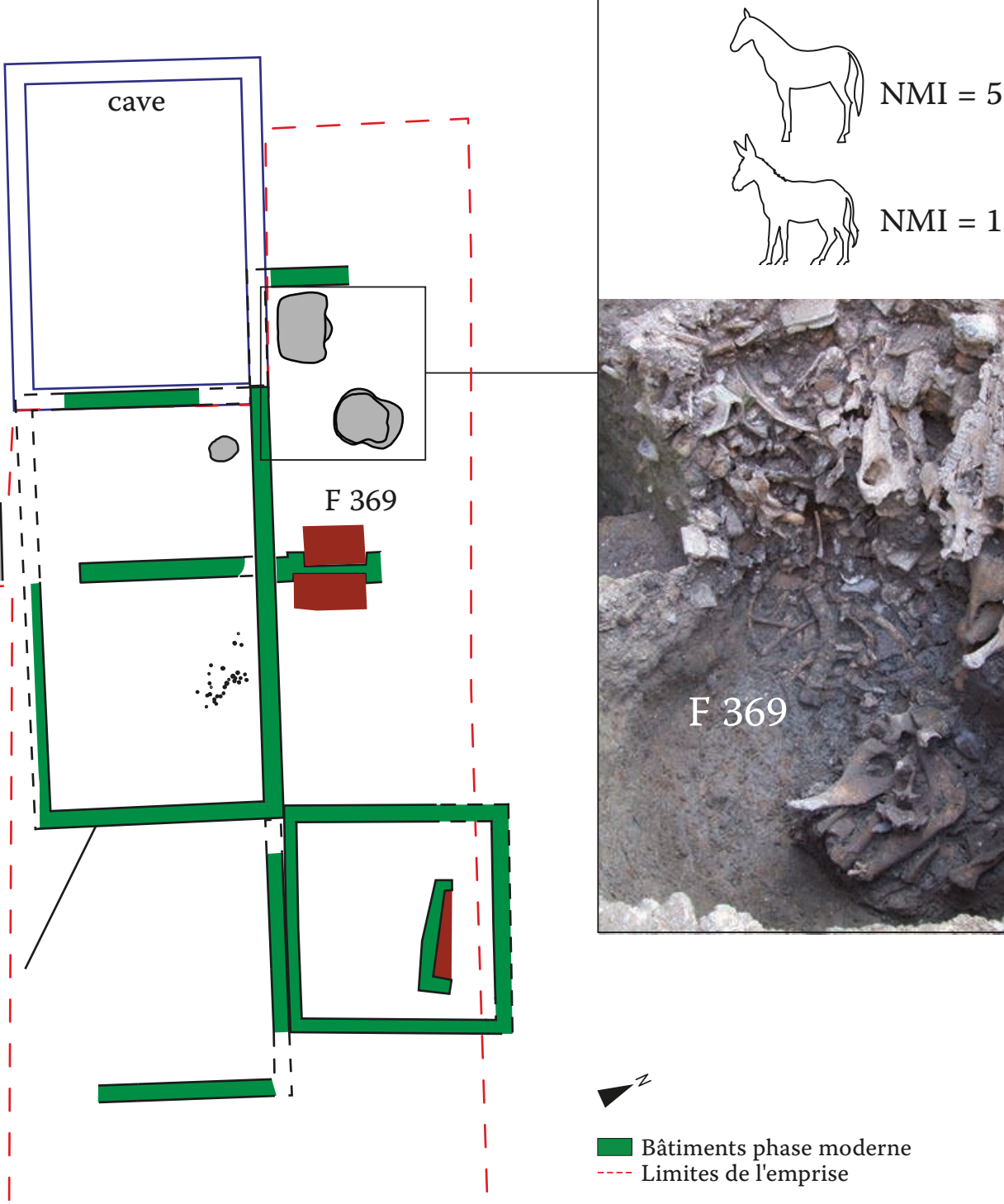

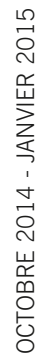

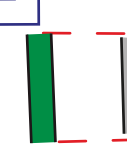




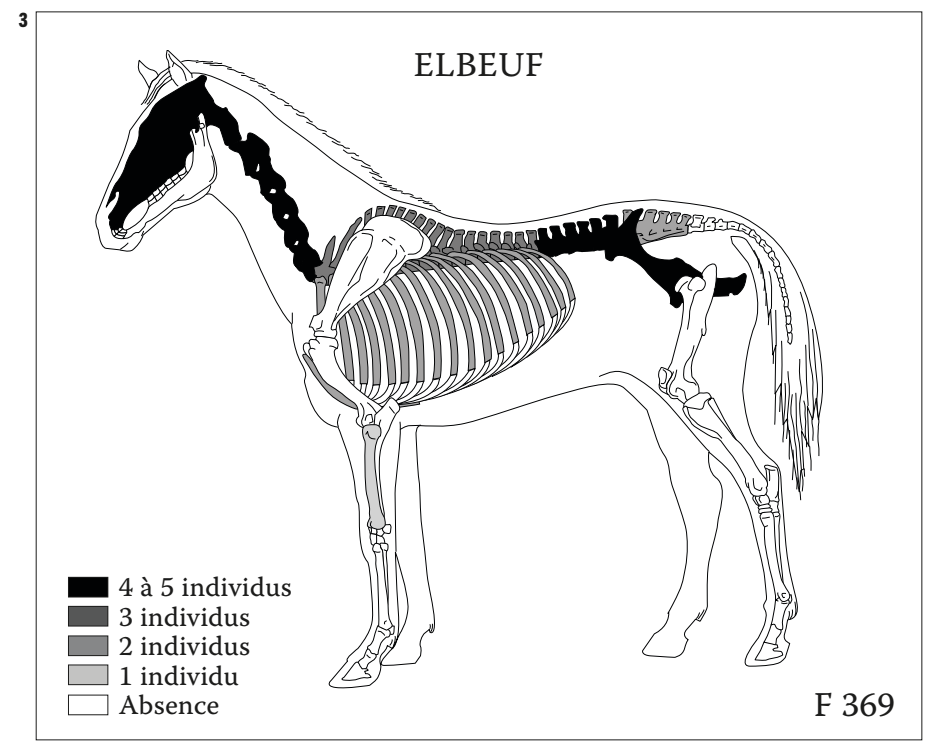

กั
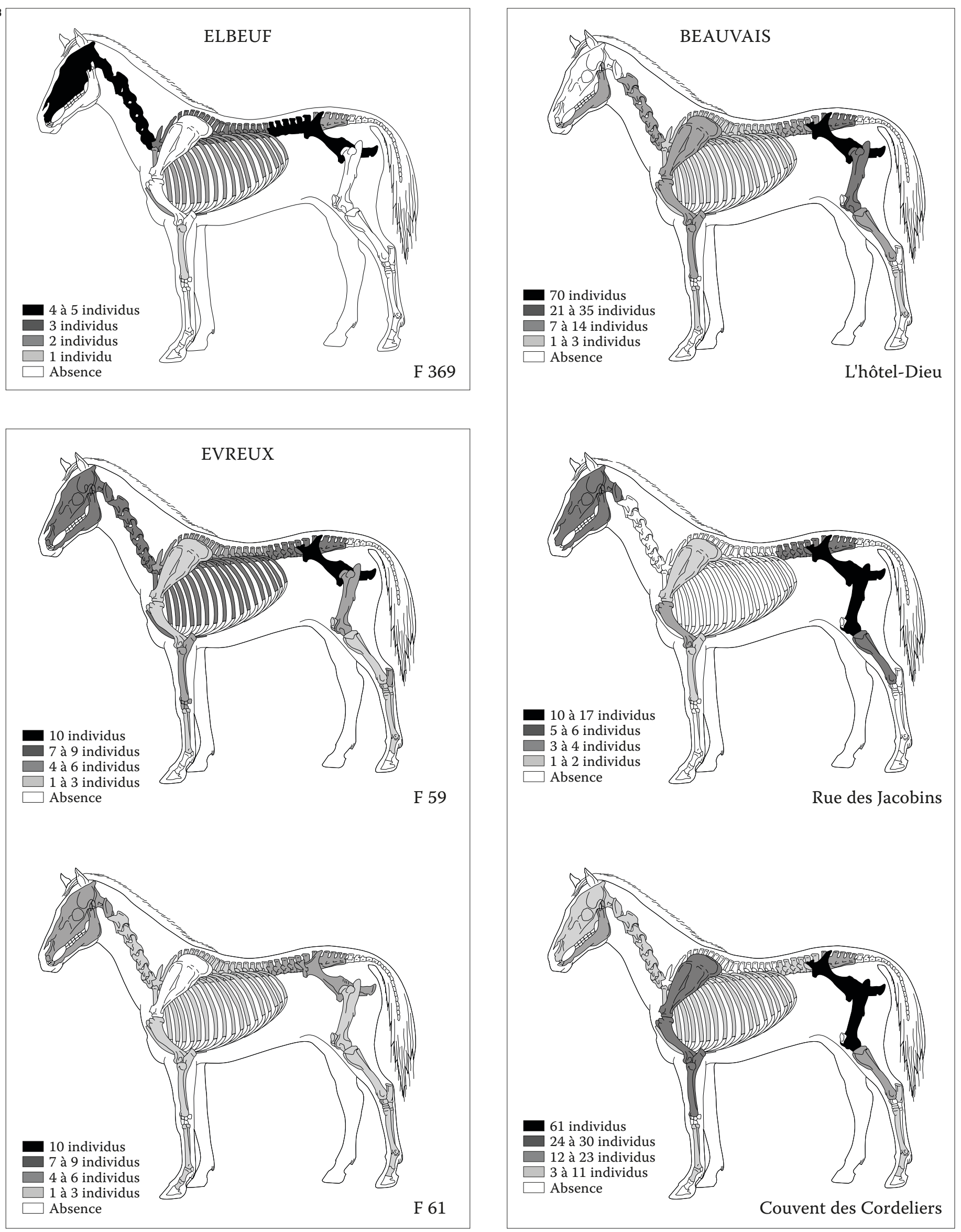
par plusieurs aspects. En premier lieu, l'abandon de ce lot osseux intervient alors que le parcellaire concerné par l'emprise de fouille se définit et se transforme en véritable quartier d'habitation (Guillot, 2013). Ainsi, la mise en place de la fosse accueillant le lot osseux étudié vient s'intercaler entre deux remaniements de cet espace en cours d'urbanisation ; elle est consécutive à l'abandon d'une double pièce à vivre, initialement aménagée au nord-ouest, lui-même causé par l'effondrement du sol [ill. 2]. L'emplacement laissé vacant se trouve ainsi rapidement accaparé, alors même que des installations mitoyennes subsistent au moment du creusement de la fosse. Par la suite, la surface occupée par la structure fossoyée fait l'objet d'un nivellement et un nouvel ensemble bâti vient sceller celle-ci.

Si la continuité de l'occupation domestique ne peut être prouvée avec certitude, le remplissage de la structure fossoyée en cause semble confirmer l'existence d'habitats proches et contemporains de son utilisation. En effet, les vestiges d'équidés exhumés à Elbeuf s'accompagnent d'une quantité non négligeable d'ossements appartenant aux animaux participant classiquement à la diète carnée (bœuf, porc caprinés domestiques, coq, etc.) : dans le premier comblement de la fosse, ayant livré l'échantillon étudié, le nombre de restes attribués aux espèces habituellement consommées demeure au moins équivalent à celui des équidés. Par ailleurs, le faciès de ces vestiges (fragmentation, stigmates liés à la découpe) renseigne

immanquablement des rejets de nature alimentaire. L'abandon des ossements d'équidés, tel qu'il s'est produit à Elbeuf, s'effectue donc dans des circonstances différentes de celles présidant habituellement à la constitution des dépôts d'équarrissage contemporains. À Évreux, les deux fosses dont proviennent les restes d'équidés, bien qu'encore incluses dans la zone intra muros, bordent la couronne non aedificandi. De même, les structures beauvaisiennes jouxtent les remparts de la cité. Cette situation topographique traduit nettement une volonté de rejeter ces éléments aux marges de la cité mais correspond également à un domaine clairement identifié comme libre d'habitats. Dans le cas du dépôt elbeuvien, cette position en périphérie de la ville se vérifie mais ne coïncide pas avec un espace déserté : le lotissement de cette zone est précisément en cours durant le $\mathrm{XVI}^{\mathrm{e}}$ siècle. En ce sens, il s'apparente plus volontiers à l'ensemble découvert dans les Jardins du Carrousel, dans l'une des parcelles d'habitation de ce faubourg naissant.

La seconde caractéristique marquante de la fosse du site d'Elbeuf tient à la nature mixte de son comblement : dans la majorité des dépôts d'équarrissage urbains et péri-urbains, la présence de vestiges appartenant à des espèces ordinairement consommées se révèle purement résiduelle. À cet égard, l'assemblage mis au jour dans l'îlot de l'Hôtel-Dieu (Beauvais) fait également figure d'exception, des restes d'espèces bouchères se mêlant aux ossements d'équidés. Néanmoins, dans ce cas comme dans celui d'Elbeuf, ces restes d'équidés relèvent manifestement d'un rejet unique et homogène : leur logique de dépôt reste strictement distincte de celle des rejets alimentaires. La rareté des dépôts combinant des rejets alimentaires à un nombre conséquent de restes d'équidés fournit d'ailleurs un indice intrinsèque du mépris cultivé par les contemporains pour la chair de ces animaux : si sa consommation n'est pas inconcevable, elle ne peut être considérée comme couramment admise et témoignerait même plutôt d'un comportement alimentaire atypique. Ainsi, en Normandie comme dans d'autres régions, l'aversion pour la chair de cheval se trouve durablement ancrée dans les mentalités du bas Moyen Âge et de lépoque moderne, du moins en contexte urbain. Elle reste une viande de « survie », comme l'atteste la description de la disette de 1693, sévissant à Rouen et sur l'ensemble du territoire normand : la misère était telle qu'on dut se résoudre à « se nourrir de chair de chevaux, de chiens et d'autres animaux » (Périaux, 1874).

Quant aux dimensions et à la composition de l'assemblage, le nombre minimum d'individus (NMI) établi sur le lot recueilli à Elbeuf n'atteint que six sujets, cinq chevaux et un âne ${ }^{3}$. La structure compte au moins deux mâles, lâne et l'un des chevaux, identifiés par la présence de canines. Par ailleurs, l'observation de la morphologie du bassin ne conduit à isoler avec certitude qu'une seule jument. L'estimation de l'âge fondée sur l'usure dentaire permet de reconnaitre un âne d'environ quinze ans et un cheval de plus de vingt ans (Cornevin, Lesbre, 1894). En revanche, l'état d'épiphysation incomplète de certains corps vertébraux trahit la présence minimale d'un individu immature, âgé de quatre ans et demi à cinq ans (Barone, 1986). Enfin, la maturité du squelette post-crânien indique encore que les autres chevaux avaient au moins cinq ans révolus.

La mise en évidence d'au moins deux individus ayant atteint un âge relativement avancé coïncide avec un profil de bêtes de réforme, les textes zootechniques médiévaux et modernes (Rusio, 1583; Serres, 160o) affirmant qu'un cheval convenablement traité et soigné devient inapte au travail entre sa quinzième et sa vingtième année. Les pathologies osseuses, marqueurs d'activité mais également de sénescence, vont d'ailleurs dans ce sens. Quant à la présence du sujet immature, elle s'apparente plutôt à une perte inopinée : le dressage débutant idéalement dans la troisième année du poulain, la force de travail de l'animal n'est ici peu ou pas rentabilisée. Cette mort prématurée peut être attribuée à l'une des multiples causes de mortalité équine, parmi lesquelles figurent l'abattage consécutif à des lésions traumatiques incurables (accidentelles ou développées du fait d'un usage inapproprié de l'animal) et les diverses maladies dont l'issue est potentiellement fatale. Encore une fois, quelques disparités existent entre l'échantillon d'Elbeuf et le schéma communément reconnu dans les dépôts d'équarrissage contemporains [ill. 3]. établie à partir des dents

jugales supérieures (Eisenmann, 1980). 

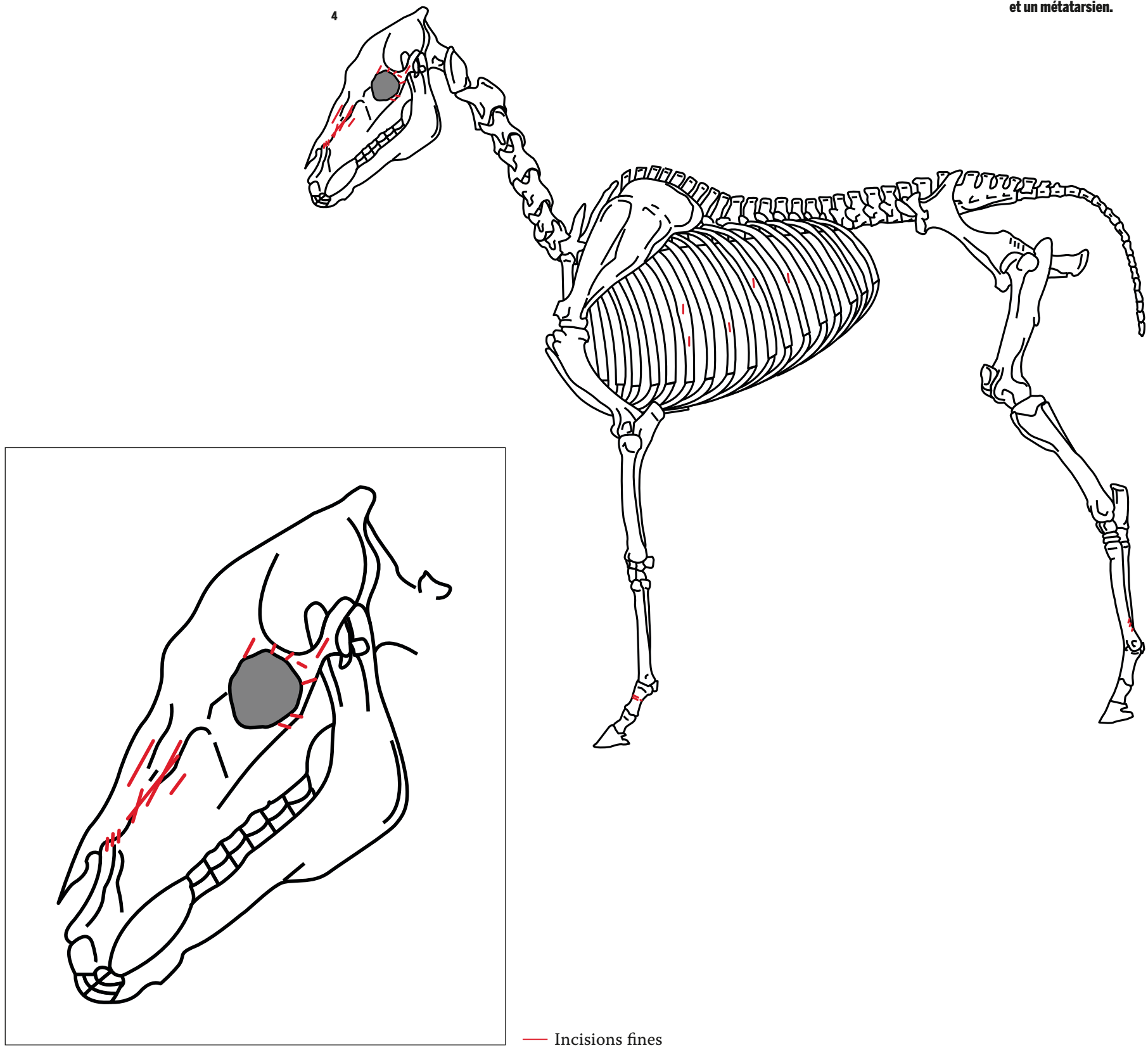
D'une part, les exemples beauvaisiens, ébroïcien et parisien concernent un nombre minimal d'individus supérieur à celui obtenu à Elbeuf. À Évreux, les fosses $\mathrm{F}_{59}$ et F61 livrent respectivement un minimum de dix et de six individus alors que la structure de la rue des Jacobins (Beauvais) en compte au moins dix-sept. La taille des échantillons recueillis sur les sites de l'Hôtel-Dieu, du couvent des Cordeliers et des Jardins du Carrousel gagne encore en ampleur, les NMI obtenus oscillant entre soixante et soixante-dix individus. Bien que le NMI n'ait qu'une valeur estimatoire (Poplin, 1976), l'activité d'équarrissage représentée par ces dépôts peut être considérée comme triplée à décuplée en comparaison de celle d'Elbeuf.

D'autre part, la présence de plusieurs vestiges d'âne, appartenant très probablement à un unique individu, s'analyse en une nouvelle particularité de l'échantillon elbeuvien. En effet, les dépôts d'équarrissage des sites septentrionaux des $\mathrm{XV}^{\mathrm{e}}-\mathrm{XVII}^{\mathrm{e}}$ siècles concernent exclusivement des chevaux, ou du moins de grands hybrides (l'unique vestige asinien repéré à Évreux reste anecdotique).

A contrario, le recrutement des animaux, en termes d'âge et de sexe, tendrait plutôt à rapprocher l'assemblage d'Elbeuf de ses homologues. Globalement, les animaux représentés dans les différents dépôts sont plus fréquemment des mâles âgés, profil cohérent avec l'utilité des chevaux et des ânes dans les milieux citadins (monte, attelage et bât). Le fait qu'il s'agisse essentiellement d'animaux de réforme, éprouvés par le travail, se remarque régulièrement dans les pathologies osseuses : les dernières thoraciques et les lombaires présentent, en particulier, des exostoses conduisant parfois à une solidarisation des vertèbres adjacentes. Enfin, les interventions pratiquées à Elbeuf sur les équidés demeurent inégalement renseignées. Ainsi, malgré la présence des crânes, aucun indice prouvant une mise à mort des animaux n'a pu être relevé. Néanmoins, la possibilité d'un abattage ne peut être totalement exclue. Il semble d'ailleurs que la décision de se séparer d'un cheval soit laissée à l'entière discrétion du propriétaire : $\mathrm{au} \mathrm{XVI} \mathrm{X}^{\mathrm{e}}$ siècle, l'obligation de supprimer les équidés pour des motifs sanitaires ne s'impose pas encore, les risques de contamination n'étant qu'imparfaitement perçus. À titre d'exemple, l'application de mesures prophylactiques en réponse aux cas de chevaux morveux, telles que l'isolement ou l'abattage des bêtes atteintes, ne se systématise que dans le courant des XVII ${ }^{\mathrm{e}}$ et XVIII ${ }^{\mathrm{e}}$ siècles (Blancou, Meslin, 200o). Or, d'origine bactérienne, la morve n'est pas seulement extrêmement contagieuse entre équidés mais se trouve encore être transmissible à l'homme (zoonose)... Les causes de ce véritable fléau, pourtant longuement décrit par l'hippiatrique au bas Moyen Âge et dans les débuts de l'époque moderne, sont imputées à des facteurs erronés : sa prévention demeure donc largement empirique et facultative.

Quant à l'exploitation des carcasses, un trait marquant du lot elbeuvien est la différence de traitement appliquée à l'âne, d'une part, et aux chevaux, d'autre part. Cependant, cette hétérogénéité de traitement ne vaut que pour cet assemblage. Sur le premier, seule la peau paraît valorisée [ill. 4], l'intention finale semble être le rejet complet de la carcasse, la présence de la majorité des os du carpe et d'un sésamoïde ${ }^{\mathbf{4}}$ plaidant pour un recouvrement alors que subsistent encore des connexions anatomiques. Néanmoins, la perte de plusieurs éléments squelettiques, et notamment de l'ensemble de la patte antérieure, indique que l'ensevelissement n'est sans doute pas immédiat. La pratique d'un dépouillement se vérifie aussi sur au moins un cheval, documentée par les stries laissées sur le crâne et les côtes. Tous les chevaux font l'objet d'un démembrement, comme l'indique l'absence quasiment systématique des portions squelettiques correspondant aux ceintures (épaules et hanches), aux membres et aux extrémités de pattes. En revanche, aucune trace ne permet d'identifier une étape ultérieure du processus d'équarrissage, telle que la récupération de matières premières (tendons, graisse, chairs, aponévroses ${ }^{\mathbf{5}}$, etc.). Enfin, la persistance de tronçons vertébraux en connexion démontre que l'ensemble du processus de décomposition ne s'est pas achevé à l'air libre. De façon générale, quelques stigmates dus aux morsures des carnassiers sur les côtes et les vertèbres signent encore un enfouissement différé du dépôt. Cependant, il s'agit plus de ponctions occasionnelles que de traces de mâchonnement intensif, ce qui suggère un accès assez bref aux ossements.

Des schémas de découpe observés dans les différents dépôts d'équarrissage se dégage l'hypothèse d'une segmentation du traitement des carcasses équines, conjuguée à l'intervention de plusieurs opérateurs spécialisés. Le premier geste concerne le dépouillement et le démembrement de l'animal, tel qu'il s'illustre dans le dépôt découvert dans les Jardins du Carrousel. Ces interventions sont documentées à Elbeuf par les stigmates de découpe, pour l'un, et les lacunes constatées dans les séries anatomiques pour l'autre. Dans un second temps, les différentes ressources monnayables sont extraites de la carcasse, lesquelles s'avèrent nombreuses si l'on en croit l'exposé de l'hygiéniste Parent-Duchâtelet, au XIX ${ }^{\mathrm{e}}$ siècle (Parent-Duchâtelet, 1827). Outre la peau et le crin, l'auteur cite le sang, la viande, les boyaux, la graisse, la corne et les os. Le prélèvement des chairs et des tendons reste archéologiquement perceptible, bien qu'avec une certaine difficulté : les pratiques d'équarrissage s'écartent des usages bouchers par l'utilisation, le plus souvent exclusive, d'une lame fine et légère. De même, les distorsions apparaissant dans les distributions anatomiques en comparaison d'une référence, soit la répartition
5. Membranes fibreuses qui enveloppent les muscles.

4. Os des extrémités 
obtenue si les carcasses étaient complètes lors de leur abandon, trahissent la sélection d'ossements spécifiques. Ainsi, le décharnement des chevaux et ces choix de pièces osseuses s'observent tant à Évreux qu'à Beauvais. Enfin, la plupart des ensembles liés à l'activité d'équarrissage témoignent d'un déficit des os de plus petites dimensions et de l'activité des carnivores qui se repaissent des carcasses abandonnées. L'enfouissement différé de ces déchets d'équarrissage semble donc être coutumier, l'aire de traitement étant nettoyée quand l'encombrement ou la nuisance olfactive l'impose.

Compte-tenu des inconvénients liés aux traitements des carcasses animales, l'existence d'une gestion encadrée des opérations d'abattage et de débitage en milieu urbain apparaîtrait légitime. Elle l'est d'autant plus dans les villes les plus imposantes, au rang desquelles figure Rouen, voisine d'Elbeuf. En ce sens, le mutisme souvent observé sur les équarisseurs dans les sources écrites se révèle instructif.

\section{Le silence évocateur des sources textuelles}

En 1502, la corporation des bouchers d'Elbeuf louait déjà à la baronnie « une chambre à mettre les chairs de la boucherie» (Maille, 1859-1863). Un document daté de 1542 précise que «les bouchers sont tenus de reposter [mettre de côté] les bestiaux qu'ils tuent » dans cet espace (Saint-Denis, 1895), ce qui semble confirmer que la modeste agglomération se trouvait effectivement dotée d'une tuerie attitrée. De même, trois tueries sont établies à Rouen dès le $\mathrm{Xv}^{\mathrm{e}}$ siècle (Ouin-Lacroix, 1850), deux convenant au traitement du grand et du petit bétail (tuerie de la Vieille-Tour et tuerie Martainville) alors que la troisième n'accueillait que les plus petits animaux (tuerie de la porte GuillaumeLion). Mais qu'en est-il de l'activité d'équarrissage? En effet, l'hypothèse d'une confusion des carcasses équines et des carcasses bouchères sur les sites de tuerie demeure hautement improbable : l'usage médiéval et moderne opère une nette distinction entre le traitement des animaux bouchers et celui des cadavres d'animaux exclus de l'alimentation, laquelle se traduit immanquablement par un éloignement de leurs lieux respectifs. En définitive, s'il existe un emplacement prévu à l'attention des équarrisseurs, aucune mention elbeuvienne ou rouennaise ne permet de le localiser : cette lacune de la documentation écrite laisse présager que l'exercice de cette " profession » ne fait souvent pas l'objet d'une réglementation stricte.

En ce sens, la comparaison avec la corporation bouchère est éclairante. En effet, l'instauration de tueries à la fin du Moyen Âge, exclusivement dédiées à l'abattage des animaux promis à une consommation humaine, ne semble pas décourager les bouchers d'officier clandestinement dans le cercle privé. Sans prétendre à une quelconque exhaustivité, plusieurs occurrences rouennaises peuvent être citées pour le seul XVI ${ }^{\mathrm{e}}$ siècle (Lormier, 1863 ; Panel, 1888 ; Ouin-Lacroix, 1850). Ainsi, le 29 décembre 1500 , les délibérations du Conseil de ville se concluent sur la nécessité que les bouchers soient désormais contraints de mener leurs bêtes aux tueries (Panel, 1888, p. 6o). Néanmoins, cette exigence s'inscrit dans une réflexion sur les moyens de conjurer un nouvel épisode de peste. De même, l'une des nombreuses épidémies de peste frappant la ville de Rouen incite l'Échiquier de Normandie à rendre plusieurs ordonnances entre 1507 et 1513 . L'un des objectifs poursuivis par les magistrats, l'assainissement des rues rouennaises, conduit à rappeler au bailli son devoir « de faire entretenir les ordonnances faictes sur le mestier des bouchers ». En conséquence, ce dernier fait publier une ordonnance rappelant qu'il «est prohibé et deffendu à tous les bouchers de cestedicte ville et autres qui s'entremettent de tuer bestes en icelle ville qu'ilz ne gettent, ne facent getter les sangz et tripailles par lesdictes rues » (Lormier, 1863).

Une mention, datée du 5 décembre 1536, indique encore qu'on réitère la consigne selon laquelle les bouchers "feront leurs massacres en la tuerie ordonnée à la Basse-Vieu-Tour» (Panel, 1888, p. 76). Enfin, une ordonnance du bailli, proclamée en 1586, commande sans équivoque aux bouchers d'abattre leurs bêtes aux tueries publiques (OuinLacroix, 1850, p. 63). La corruption des rues rouennaises occasionnée par l'activité bouchère s'avère donc régulièrement évoquée. De même, les ordonnances visent les «conrieurs » [= corroyeurs], cordonniers et autres «fondeurs de graisse », contribuant à l'empuantissement de l'atmosphère des villes (Lormier, 1863). Enfin, toujours à Rouen, les métiers associés au travail du cuir se concentrent tous initialement sur le cours de la Renelle : ce n'est que secondairement que les mégissiers éloignent spontanément leurs installations sur l'Aubette (la Maresquerie), au cours du Xv $\mathrm{Xv}^{\mathrm{e}}$ siècle. Au siècle suivant, les plaintes formulées par les riverains contre les tanneurs suscitent des tentatives pour reloger ces derniers sur le Robec, tentatives contrariées par la récalcitrance des intéressés (Lormier, 1863 ; Panel, 1888).

Cette tendance à maîtriser ou écarter les activités les plus polluantes des villes, illustrée ici à Rouen, procède d'un mouvement plus général et se heurte partout à une constante résistance. Pourtant, encore une fois, la sordide besogne des équarisseurs n'est jamais citée dans les textes elbeuviens ou rouennais, sauf à les reconnaître occasionnellement dans les fondeurs de graisse. Un litige opposant des riverains à deux écorcheurs, pratiquant régulièrement l'équarrissage, va en ce sens : l'exposé des griefs contenus dans l'arrêt du parlement de Paris rendu en 1707 cite la pestilence des « graisses $\mathcal{E}$ huiles de chevaux nouvellement fondües » et les «peaux puantes » stockées dans la maison des accusés (Nupied, 1757). En définitive, le mutisme suggère encore la relative discrétion de cette occupation et, vraisemblablement, son caractère secondaire aux yeux des autorités. 
Ce long mais nécessaire développement sur les métiers participant à la gestion des produits issus des carcasses animales démontre, certes, l'impuissance des témoignages écrits à saisir la réalité des pratiques d'équarrissage, mais surtout l'inexistence d'un cadre normatif définissant leur exercice dans beaucoup de milieux urbains ou péri-urbains. Paris, pourvue d'au moins une « escorcherie aux chevaux » avant 1404 (ParentDuchâtelet, 1827), reste un épiphénomène.

Plusieurs arguments peuvent être avancés pour comprendre cet apparent désintérêt. En premier lieu, le nombre de bêtes que les bouchers ont à traiter est sans commune mesure avec celui des équarisseurs, occasionnels ou professionnels. Ainsi, si les statuts corporatifs des bouchers elbeuviens ne nous sont pas parvenus, ceux de Rouen (Ouin-Lacroix, 1850) stipulent que l'introduction de viande déjà débitée dans l'enceinte de la cité est proscrite, ce pour préserver le monopole dont jouissaient les bouchers urbains sur la vente de l'essentiel des viandes : l'approvisionnement de Rouen s'effectue principalement sous forme de bétail sur pied, animaux insusceptibles d'être cédés aux forains une fois pénétrés dans la ville. À titre indicatif, la consommation hebdomadaire rouennaise satisfaite par les bouchers équivaut à environ 250 bœufs ou vaches et à 4000 moutons en 1520 (le décompte excluant porcs et veaux). Empiriquement, le nombre d'équidés ne peut qu'être nettement inférieur : aux bêtes possédées par les particuliers s'ajoutent notamment celles des loueurs de chevaux et plus tardivement de carrosses, dont la liberté d'industrie laisse supposer le nombre relativement restreint. Quant aux charretiers rouennais, ils étaient en état d'atteler cent dix-neuf véhicules en 1730 (OuinLacroix, 1850). Bien qu'ultérieure de deux siècles, cette mention laisse présager un effectif plus modeste encore à l'époque qui nous intéresse. Même en prenant en considération les chevaux de poste et ceux dévolus aux coches, voitures et messageries, l'effectif des équidés réformés ou morts accidentellement demeure nécessairement dérisoire en comparaison de celui des animaux de boucherie abattus. Par ailleurs, le traitement de ces quelques carcasses équines ou asiniennes ne peut rivaliser avec la nuisance olfactive produite par les tanneries rouennaises, qui regroupaient près de neuf cents bâtiments en 1581 (Ouin-Lacroix, 1850).

Une autre raison de la discrétion de la documentation juridique tient sans doute aux enjeux sanitaires associés à l'activité bouchère. En effet, l'insistance des autorités à astreindre les bouchers à fréquenter les tueries ne procède pas uniquement d'un souci de salubrité : l'abattage et les premiers apprêts des carcasses sur un lieu public permettent de garantir des produits carnés sains. Ce dispositif venait sans doute utilement doubler la sanction frappant les marchands présentant des bêtes malades sur la place du marché, comme à Rouen : outre l'amende infligée au contrevenant, l'animal se voyait abattu sur le champ et ses chairs jetées dans le fleuve (OuinLacroix, 1850). Dans la mesure où les équidés ne participent pas à la diète carnée humaine, du moins en principe, leur état général et le devenir de leurs cadavres n'engendrent logiquement pas les mêmes inquiétudes.

Enfin, une gestion domestique des animaux consommables ou non subsiste en milieu urbain. En effet, la pratique d'un élevage privé ou « semiprivé » dans les enceintes urbaines médiévales et modernes n'est plus à démontrer. À titre d'exemple, les termes des procès-verbaux de délibérations du Conseil de ville, datés de 1498, confirment la divagation d'oies et de porcs dans les rues de Rouen (Panel, 1888, p. 6o). De même, en 1536, il est mis un coup d'arrêt à l'élevage des "pourceaux, oysons, connins [= lapins] et coullons [= pigeons] » par le prieuré de la Madeleine et la commanderie de Saint-Antoine (Panel, 1888, p. 61). Cet état de fait rend plausible la pérennité de l'exercice boucher familial et communautaire et, en conséquence, la relative tolérance à l'égard de leurs auteurs. Par analogie, il est vraisemblable que les particuliers n'aient pas toujours recours à un opérateur professionnel pour exploiter un cheval perdu.

Le dépôt d'équarrissage exhumé à Elbeuf se singularise par sa petite envergure et la mixité des rejets mêlant déchets alimentaires et restes d'équidés. Par ailleurs, la présence d'une carcasse d'âne parmi les quartiers de chevaux contribue à l'originalité de l'ensemble. L'influence du lieu de traitement, vraisemblablement proche de celui du dépôt, peut être invoquée pour éclairer ces caractéristiques. La considération selon laquelle l'étendue et la densité du tissu urbain conditionnent la taille et la mono-spécificité des dépôts d'équarrissage reste alors cohérente avec l'hypothèse d'une augmentation de l'importance des dépôts au cours du temps : le niveau de développement urbain serait le critère déterminant, tant dans l'espace que dans le temps. Cependant, si l'importance relative de la ville d'Elbeuf contribue à expliquer ces particularités, elle n'y suffit pas : la cité ne rivalise pas avec Paris ou Rouen mais sa puissance démographique ne diffère sans doute pas fondamentalement de celle d'Évreux. En revanche, la position du dépôt dans un quartier d'habitation en devenir est propice à l'improvisation d'une fosse d'équarrissage. L'abandon des restes d'équidés correspond vraisemblablement à un événement ponctuel et relève plus d'une gestion «semiprivée » des carcasses que d'une activité de plus grande ampleur. En ce sens, le fait que la structure recevant ces déchets ait également vocation de dépotoir alimentaire, dès l'origine ou à l'issue d'une reconversion immédiate après le rejet, démontre une certaine prégnance de la sphère domestique dans ce secteur.

Par ailleurs, si l'activité et la spécialisation croissantes des équarisseurs se dessinent au cours 


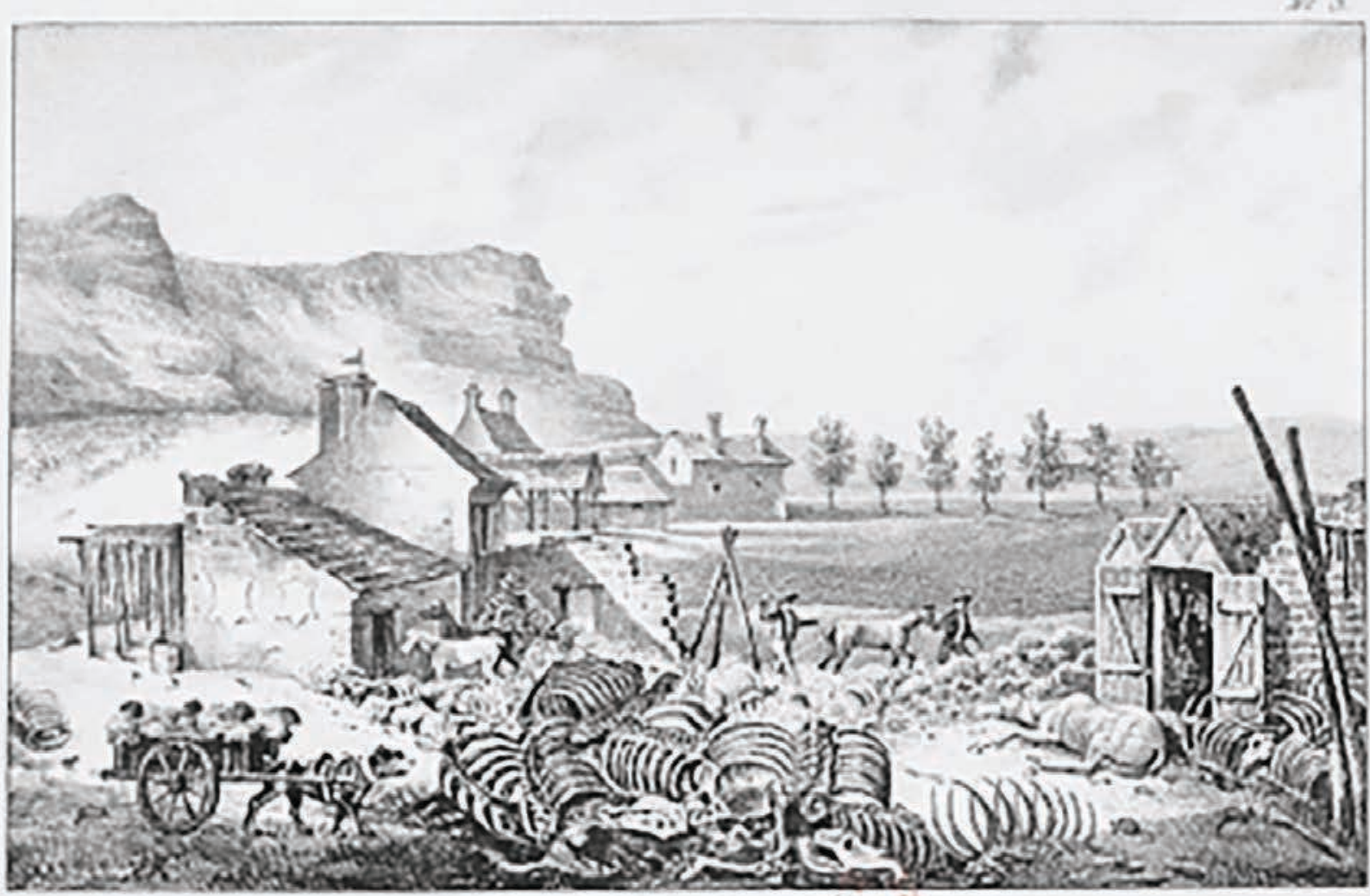

5. Au xixe siècle, Parent-

plusieurs reprises la voierie

de Montfaucon où se

situe alors l'équarrissa

II loue la dextérité

des ouvriers chargés de

ce dur labeur et décrit

minutieusement les

différentes opérations

liées à cette activité. Su cette figure, l'illustrateur a représenté l'intérieur d'un clos où se trouvent accumulées, en grand nombre, les carcasses équines. Sur le pignon du bâtiment de gauche sont également représentées des peaux de grands chiens mises à sécher. 
du Moyen Âge et de l'époque moderne, il n'en reste pas moins que cette «profession » garde une dimension marginale. La rareté des normes bornant l'exercice de ce travail et l'apparente inexistence de lieux qui lui soient officiellement dédiés donnent le sentiment d'une certaine permissivité dans les pratiques d'équarrissage. Remarquons d'ailleurs que le terme " équarrissage ", ici employé par commodité, constitue un anachronisme pour la période considérée : le terme ne commence à s'appliquer aux chevaux et à leurs « écorcheurs » que dans le courant du XVIII ${ }^{\mathrm{e}}$ siècle [ill. 5]. En effet, cette activité particulière se trouve auparavant assimilée aux travaux d' «écorcherie », appellation plus générique et qui n'est d'ailleurs pas sans poser quelques difficultés sémantiques.
Néanmoins, le caractère relativement officieux des travaux d'équarrissage n'implique pas nécessairement que leurs auteurs soient affranchis de la nécessité de reléguer les ordures qu'ils génèrent aux marges de la ville. Cet impératif est d'abord dicté par l'esprit général des prescriptions, enjoignant à tout habitant de ne pas abandonner ses immondices sur la voirie. Il tient ensuite au bon sens et à la volonté de s'épargner les foudres du voisinage : la proximité d'un lieu d'équarrissage étant nécessairement perçue comme une véritable calamité, les abus ne manquaient pas d'entraîner des plaintes. En définitive, le dépôt d'équarrissage d'Elbeuf traduit une situation qu'on pourrait qualifier d'intermédiaire, représentative du lien étroit entre mode de gestion et contexte de rejet. En l'occurrence, le secteur semi-urbain de la parcelle et les dimensions « familiales » de l'activité déterminent sans doute une tolérance résignée.

\section{Références bibliographiques}

LORMIER C. (ÉD.), 1863, Ordonnances contre la peste et autres ordonnances concernant la salubrité publique dans la ville de Rouen, rendues par la cour de l'Échiquier de 1507 à 1513 [...], Rouen, Impr. de Henry Boissel, VIII-22 p.

Nupied N. (éd.), 1757, Journal des principales audiences du parlement, avec les arrêts qui y ont été rendus et plusieurs questions et règlemens placés selon l'ordre des temps, depuis l'année 1700 jusqu'en 1710, Paris, Compagnie des Libraires associés, vol. 5 , XXXI68o-388 pp.

OuIN-LACroix C., 1850, Histoire des anciennes corporations d'arts et métiers et des confréries religieuses de la capitale de la Normandie, Rouen, Imprimé par Lecointe frères, XII-763 p.

PANel G., 1888, Préoccupations municipales pour l'hygiène et la santé publiques à Rouen : résumé historique de 1389 à 1870, Rouen, Imprimerie de Espérance Cagniard, 84 p.

Parent-Duchâtelet A.-J.-B., 1827, Recherches et considérations sur l'enlèvement et l'emploi des chevaux morts et sur la nécessité d'établir à Paris un clos central d'écarrissage, tant pour les avantages de la salubrité publique que pour ceux de l'industrie manufacturière de cette ville, Paris, Bachelier, 124 p.

Rusio L., 1583, La Mareschalerie de Laurent Ruse, où sont contenuz remèdes très singuliers contre les maladies des chevaux, avec plusieurs figures de mors; en laquelle y avons adjousté un autre traicté de remèdes [...], (rééd. Paris, G. Auvray, 1583), 112 p.

Serres O. de, 16oo, Le Théâtre d'agriculture et mesnage des champs, d'Olivier de Serres, seigneur du Pradel, (Nouvelle édition conforme au texte, augmentée de notes et d'un vocabulaire, Paris, Librairie de Madame Huzard / Société d'agriculture du département de la Seine, 1804-1805), 2 vol., 672-948 p.
Arbogast R.-M., Clavel B., Lepetz S., Méniel P. YVINEC J.-H., 2002, Archéologie du cheval: des origines à la période moderne en France, Paris, Éditions Errance, 127 p.

Barone R., 1986 ( 3 éd.), Anatomie comparée des mammifères domestiques, t. 1 : ostéologie, Paris, Vigot, XIX-761 p.

Blancou J., Meslin F.-X., 200o, « Brefs rappels sur l'histoire des zoonoses ", Revue scientifique et technique de l'Office international des épizooties, vol. 19, fasc. 1, p. 15-22.

Clavel B., Robin O., 2012, « Les animaux en ville. Des squelettes médiévaux et modernes à Amiens ", Archéopages, $\mathrm{n}^{\circ}$ 35, p. 48-51.

Cornevin C., Lesbre F.-X., 1894, Traité de l'âge des animaux domestiques d'après les dents et les productions épidermiques, Paris, J.-B. Baillière, VIII-462 $\mathrm{p}$.

EISENMANN V., 1980, Les Chevaux (Equus sensu lato) fossiles et actuels : crânes et dents jugales supérieures, Paris, Éditions du Centre national de la recherche scientifique (Collection Cahiers de Paléontologie), $186 \mathrm{p}$.

Guillot B., 2013, «Elbeuf. 57 rue Guynemer », Bilan scientifique de la région Haute-Normandie, p. 58-62.

GuILlOT B., à paraître, Elbeuf, 57 rue Guynemer, rapport d'opération, Inrap-SRA Haute-Normandie.

LEPETZ S., 1991, « Les restes animaux des fouilles de l'Hôtel-Dieu à Beauvais (Oise) ", Revue Archéologique de Picardie, n 3-4, p. 273-292.
MAILLE P., 1859-1863, Recherches sur Elbeuf, Elbeuf, Bureau de l'imprimerie, 3 vol.

PÉRIAUX N., 1874, Histoire sommaire, et chronologique de la ville de Rouen, de ses monuments, de ses institutions, de ses personnages célèbres, Rouen, Lanctin \& Métérie, 706 p.

Poplin F., 1976, « À propos du nombre de restes et du nombre d'individus dans les échantillons d'ossements ", Cahiers du centre de recherches préhistoriques de l'Université de Paris I, 5, p. 61-73.

Poplin F., 2013, "Préface», in Auxiette G. et Ménie P. (éd.), Les dépôts d'ossements animaux en France, de la fouille à l'interprétation, Actes de la table ronde de Bibracte, 15-17 oct. 2012, Montagnac, M. Mergoil, 286 p.

Rodet-BelARbi I., 1993, « Deux activités artisanales d'un faubourg parisien des $\mathrm{XV}^{\mathrm{e}}$ et $\mathrm{XVI}^{\mathrm{e}} \mathrm{s}$. d'après les ossements animaux ", Anthropozoologica, n ${ }^{\circ}$ 17, p. 11-20.

Rodet-Belarbi I., Jouanin G., Clavel B., à paraître, « La gestion des restes d'équidés en ville et à la campagne en France au Moyen Âge et au début de l'époque Moderne ", in Vie et mort du cheval: des pratiques médiévales aux traditions camarguaises, Actes du $\mathrm{X}^{\mathrm{e}}$ congrès de la Société d'Archéologie Médiévale (Arles, 3-5 mai 2012).

SAINT-Denis H., 1895, Histoire d'Elbeuf, t. II (de 1450 à 1630), Elbeuf, Imprimerie H. Saint-Denis, 598 p. 